\title{
Atomic photoionization in changing plasma environment
}

\author{
T. N. Chang ${ }^{1}$ and T. K. Fang ${ }^{2}$ \\ ${ }^{1}$ Department of Physics and Astronomy, University of Southern California, Los \\ Angeles, CA 90089-0484, U.S.A. \\ ${ }^{2}$ Department of Physics, Fu Jen Catholic University, Taipei, Taiwan, 242, ROC
}

We present a detailed theoretical study on the photoionization of one- and twoelectron atoms subject to external changing plasma environment based on the DebyeHückel model. Specifically, by examining the migration of the bound excited states into the continuum in the presence of plasma, our investigation has led to resonance-like photoionization structures immediately above the ionization thresholds for $H, \mathrm{H}^{-}$, and $\mathrm{He}$ atoms. For $\mathrm{He}$, unlike the usual Fano-Beutler resonances due to the configuration mixing between the doubly excited components and the singly ionization channel with different $\ell_{1} \ell_{2}$ angular momenta combination in the final state wavefunction, these plasma induced resonance-like structures result from the mixing of the quasi-bound and continuum components of the same $\ell_{1} \ell_{2}$ combination in the final state function. With a number of specific examples, we will show that the general features of these spectra could be linked directly to the overlap at small $r$ between the effective wave functions of the outgoing ionized electron and the atomic electron in its initial states.

PACS number(s); 32.80.Fb, 32.80.Gc, 32.20.Jc, 52.25.Os 


\section{INTRODUCTION}

Recent theoretical studies of atomic processes in plasma environment [1-10] have attracted considerable renewal interests following a number of earlier works [11-13] based on the application of the Debye-Hückel model [14] for a classical electron-ion collisionless plasma under thermodynamical equilibrium. Nearly all such theoretical estimations [110] were carried out with a screened Coulomb potential $V_{s}$ (or, the Yukawa potential) for an atomic electron in the vicinity of a nuclear charge $Z$ subject to a one-electron Hamiltonian, i.e.,

$$
h_{o}(r ; D)=\frac{p^{2}}{2 m}+V_{s}(r ; D) \text { and } V_{s}(r ; D)=-\frac{Z e^{2}}{r} e^{-r / D},
$$

where $p$ is the momentum of the electron and the parameter $D$ is known as the DebyeHückel length (or Debye length). The Debye length characterizes the screening of the nuclear charge experienced by the atomic electron due to the plasma in the outer region of an atom and is given in Bohr radius $a_{o}$ by (see also, e.g., Eq. (2.5) of [13])

$$
D=1.304 \times 10^{8}(T / n)^{1 / 2} a_{o},
$$

where $T$ and $n$ are the plasma temperature (on Kelvin scale) and density (in $\mathrm{cm}^{-3}$ ), respectively. Or, alternatively, it can be expressed in the unit of $\mathrm{cm}$ by $D=6.90(T / n)^{1 / 2}$. The value of $D$ ranges over many orders of magnitude, i.e., from $10^{-2} \mathrm{~nm}$ for the plasma in solar core to over $10^{4} \mathrm{~m}$ for the ones in intergalactic medium. Since the plasma temperature usually varies only 3 to 4 orders in magnitude (e.g., from $10^{2}{ }^{\circ} \mathrm{K}$ to $10^{6}{ }^{\circ} \mathrm{K}$ ), the value of $D$ (or, the degree of penetration of the ion-electron gas into the atom) depends more on the variation of the plasma density.

For the low-density plasmas such as the ones in interstellar medium or even for those generated in low power gas discharge, the Debye length $D$ may be a few orders of magnitude larger than the size of the atoms and should have little effect on the atomic processes. Since the plasma frequency for electron (i.e., $\omega_{p} \sim n^{1 / 2}$ ) is also relatively small and the period of plasma oscillation is substantially longer than the time scale of a typical atomic process, the plasma environment effect on atomic transition is also expected to be minimal. On the other hand, for the high density plasma system such as the ones in the Sun and Sun-like stars, the Debye length is of the order of $10^{-11} \mathrm{~m}$. Consequently, the effect of the Debye screening could change significantly the atomic transitions for the astrophysical relevant elements. That, in turn, could have serious implication to the opacity data, which is of critical importance to the development of high quality equation of state for such systems [15]. In fact, both the commonly applied equation-of-state models (MHD and OPAL) include the leading Coulomb correction 
(i.e., the Debye-Hückel term) to ideal-gas thermodynamics in the current standard solar models [16].

Following the Debye-Hückel original approximation, the potential $V_{o}(r)$ due to an electron-ion plasma at a distance $r$ far from a force center (e.g., an atomic nucleus) outside an inner Debye sphere of radius $A$ can be derived from the Gauss' law

$$
\nabla^{2} V_{o}(r)=-\frac{\rho(r)}{\epsilon_{p}}, \quad r \geq A,
$$

where $\epsilon_{p}$ is the dielectric constant of the electron-ion gas and $\rho$ is its total charge density at $r$. Assuming a charge density of $\rho_{o}$ at $r=\infty$ with a zero potential $V_{o}(r=\infty)=0$ and following the Boltzmann distribution, the charge densities of the positive charge $q$ and the negative charge $-q$ at $r$ could be expressed as $\rho_{+}(r)=\rho_{o} e^{-q V_{o}(r) / \kappa T}$ and $\rho_{-}(r)=\rho_{o} e^{+q V_{o}(r) / \kappa T}$, respectively, where $\kappa$ is the Boltzmann constant and $T$ the absolute temperature. The net total charge density at $r$ is then given by

$$
\rho(r)=\rho_{o}\left(e^{-\frac{q V_{o}(r)}{\kappa T}}-e^{\frac{q V_{o}(r)}{\kappa T}}\right)=-2 \rho_{o} \sinh \left(\frac{q V_{o}(r)}{\kappa T}\right) .
$$

Assuming, in addition, that the potential energy is relatively small comparing to the kinetic energy, Eq. (3) for the potential $V_{o}(r)$ in the outer region of the Debye sphere could then be approximated by the linear Poisson-Boltzmann equation

$$
\nabla^{2} V_{o}(r)=\left(\frac{1}{D^{2}}\right) V_{o}(r), \quad r \geq A,
$$

where $D>A$ is the Debye length given by Eq. (2) and defined in terms of the charge density $n\left(\sim q \rho_{o}\right)$ and the temperature $T$ outside the Debye sphere. The potential inside the Debye sphere can also be derived from the Gauss' law and takes the form of

$$
V_{i}(r)=-\frac{Z e}{r}+\text { Constant }, \quad r \leq A
$$

with a nucleus charge $Z$ located at $r=0$. By matching the two potentials $V_{o}$ and $V_{i}$ and their first-order derivatives at $r=A$, one gets $[12,13]$

$$
V_{s}(r ; D)= \begin{cases}V_{i}(r)=-Z e^{2}\left(\frac{1}{r}-\frac{1}{D+A}\right), & r \leq A \\ V_{o}(r)=-Z e^{2}\left(\frac{D e^{A / D}}{D+A}\right) \frac{e^{-r / D}}{r}, & r \geq A .\end{cases}
$$

In the limit when $A \rightarrow 0$, Eq. (7) reduces approximately to the screened Coulomb potential employed in most of the recent theoretical atomic calculations. Clearly, the Debye model breaks down when $D \rightarrow A$. An alternative screened Coulomb potential for Debye screening with four parameters was introduced by Wang and Winkler [8] in 
their theoretical calculation. For the one-electron atom, the most interesting feature resulting from the screened Coulomb potential is the disappearance of the bound state as $D$ becomes smaller than the corresponding critical screening length of that particular state (see, e.g., Tables III and IV of [11]). In other words, the potential can only attract a decreasingly finite number of bound states below the ionization threshold as $D$ decreases [11-13]. We should point out that any estimate of the plasma effect on atomic process based on Debye-Hückel model should be limited to Debye lengths that are sufficiently longer than the radius $A$ of the inner Debye sphere, i.e., the average size of the plasma-free atoms.

In addition to the solar plasma discussed earlier, for the laser-produced dense plasma at an electronic temperature of a few hundred $\mathrm{eV}$ and a density of $10^{22}-10^{24} \mathrm{~cm}^{-3}$ $[17,18]$, the Debye length would be comparable to the size of the atoms. Although the laser-produced plasma is far from a thermodynamically equilibrium system, a detailed theoretical estimate based on the Debye-Hückel model may help to better understand the physics behind the observed spectra, particularly the shift of the spectral lines. Qualitatively, the shift in energy levels due to the increase in plasma density (or, as $D$ decreases) would then lead to a red-shift in the resulting spectral lines in the emission spectrum. Indeed, such shifts were observed in the laser-produced high density plasmas near the surface of the solid graphite targets [17].

An equally interesting question would then be what happen to the bound-bound transitions (i.e., the emission lines) when the upper excited states moves into the continuum. Some of the recent studies on photoionization and electron-atom impact ionization have led to a fairly general feature, i.e., the presence of narrow resonance-like structure immediately above the ionization threshold and its transformation to broad continuum structure as the Debye length decreases, or, when the plasma effect increases $[4,5]$. This general feature was attributed plausibly either as the shape resonance or a transformation of Feshbach resonance into shape resonance. It is also interesting to note that there is a sharp increase of the cross sections from a value of zero at the threshold to the peak cross section, in contrast to the known monotonically decreasing photoionization cross sections for the plasma-free hydrogen as energy increases [19]. It is the main purpose of this paper to show that such features could be easily and directly linked to the overlap of the effective one-electron wavefunctions between the bound electron initially and the outgoing electron in the continuum. We will also present in detail the migration of the photoionization spectra of two-electron systems such as $\mathrm{He}$ and $\mathrm{H}^{-}$and show that the absence of bound excited state for plasma-free $H^{-}$will lead to spectra that are very different from the resonance-like structures for $\mathrm{H}$ or $\mathrm{He}$. 


\section{PHOTOIONIZATION OF ONE-ELECTRON ATOM}

By using the discretized B-spline based atomic orbits, the photoionization cross section $\sigma$ from an initial state $I$ to a final continuum $F$ with an outgoing electron of momentum $k$ could be expressed in terms of the oscillator strength $f_{F I}$ by the expression $[20,21]$

$$
\sigma=\frac{4 \pi \alpha}{k A_{o}^{2}} f_{F I},
$$

where $\alpha$ is the fine structure constant and $A_{o}$ is the amplitude of the outgoing oneelectron oscillating wavefunction. We should note that the usual per unit energy normalization constant $(2 / \pi k)^{1 / 2}$ is replaced by $A_{o}$ for the B-spline based atomic wavefunctions confined in a sphere of radius $R$. For the one-electron atoms, the one-particle radial wavefunctions $\chi_{\nu \ell}(r)$ subject to the potential $V_{s}$, either in the form of Eq. (1) or Eq. (7), are generated by the one-electron Hamiltonian given by Eq. (1), i.e.,

$$
h_{o}(r ; D) \chi_{\nu \ell}=\epsilon_{\nu \ell} \chi_{\nu \ell}
$$

where $\ell$ is the orbital angular momentum and $\epsilon_{\nu \ell}$ is the energy eigenvalue. For the outgoing ionized electron, $\nu$ represents the energy $\epsilon=k^{2}$ in $R y$ unit. The oscillator strength $f_{F I}$ and its corresponding photoionization cross section are determined by the one-electron dipole radial matrix $<\chi_{n \ell}|d| \chi_{k \ell^{\prime}}>$ of the dipole operator $d$, either in length or velocity approximation, between the wavefunctions of the initial state $\chi_{n \ell}$ and the final state $\chi_{k \ell^{\prime}}$. Qualitatively, it is clear that the greater the overlap between $\chi_{n \ell}(r)$ and $\chi_{k \ell^{\prime}(r)}$ the larger the cross section. In the present calculation, the numerical accuracy of our calculation is assured as the length and velocity results of the cross section are in agreement to 4 digits or better.

Figure 1 presents our theoretical results on the variation of the hydrogen photoionization spectra corresponding to the $1 s \rightarrow 4 p$ transition as the Debye length decreases from the critical screening length of the $4 p$ state, which is slightly greater than $25 a_{o}$. We have chosen the migration of the $1 s \rightarrow 4 p$ transition as our example to assure that the range of the Debye lengths is sufficiently large compared to the size of the hydrogen atom in its $1 s$ ground state, so that the Debye-Hückel model is a reasonable approximation to emulate the outside plasma environment. The top plot of Fig. 1 confirms the general feature suggested by the recent theoretical results $[4,5]$ discussed earlier, i.e., from a narrow resonance-like structure at a $D$ value close to the critical screening length to a broad continuous spectrum as $D$ decreases. The bottom plot of Fig. 1 shows the redshift from the $4 p \rightarrow 1 s$ emission line, now in terms of a narrow resonance-like absorption

line near $989.5 \AA$ as the $4 p$ state moves into the continuum due to the increase of plasma 
effect. This shift is significant compared to the corresponding plasma-free Lyman- $\gamma$ line at $972.5 \AA$.

For a plasma-free hydrogen atom, the radial part of the one-particle wavefunctions at small distance $r$ from the nucleus for the outgoing $k p$ electron are essentially the same until they are sufficiently away from the nucleus. The top plot of Fig. 2 shows such wavefunctions at a number of momenta $k$. As expected, they all reach their first local maxima and the subsequent zeros at about the same values of $r$ as shown. In other words, the penetration of the wavefunctions at small $r$ depends little on the energy (or momentum) of the outgoing electron. For photoionization, the difference in the $k p$ wavefunctions at large distance, in fact, has little effect on its cross section. The slight difference in the first loop of the wavefunctions and its overlap with the wavefunctions of the initial $1 s$ electron (with its maximum at $r=a_{o}$ shown in the bottom plot of Fig. 2) dictate entirely the photoionization spectrum. Consequently, as we pointed out earlier, the photoionization spectrum for plasma-free hydrogen is known to decrease monotonically as the energy increases [19].

For the screened Coulomb potential with $D=24.5 a_{o}$ (i.e., at a $D$ value slightly less than the critical screening length for the $4 p$ state), the radial wavefunctions shown in Fig. 3 for the outgoing electron at energies near the threshold behave very differently from that shown in Fig. 2. Although the local maxima and their subsequent zeros for the first loop of all three wavefunctions $\chi_{k p}$ near the origin stay at the same $r$ as the ones shown in Fig. 2, their magnitudes are substantially different as $k$ increases. At $k=0.014953$, the magnitude of its first loop is almost zero, representing a very small penetration of the ionized electron into the inner region of the atom due to its relatively small kinetic energy. At a slightly higher energy near $k=0.034244$, our calculation leads to a quasibound radial wavefunction with a relatively small amplitude for the outside oscillating wavefunction. As the energy increases further, $\chi_{k p}$ with $k=0.053371$ behaves more like the plasma-free ones shown in Fig. 2. Since the radial wavefunction of the initial $1 s$ electron is mostly confined at $r$ less than $5 a_{o}$, the dipole matrix element is essentially determined by the overlap between the first loop of the $\chi_{k p}$ and $\chi_{1 s}$. Therefore, with a magnitude of near-zero first loop at $k=0.014953$, it is easy to conclude a nearzero threshold cross section as shown in Fig. 1. As the energy increases to that for the quasi-bound state at $k$ close to 0.034244 , the overlap between $\chi_{k p}$ and $\chi_{1 s}$ is at its maximum and thus the peak of the sharp narrow resonance-like structure. At even higher energy when the radial wavefunctions behave like the plasma-free atom, a monotonically decreasing cross section such as the one shown in Fig. 1 is expected. Our calculation shows progressively a more diffused quasi-bound radial wavefunction with decreasing $D$, thus, the broadening of the resonance-like structure. Eventually, the narrow resonancelike structure evolves into a continuous spectrum as shown in Fig. 1. Our explicit 
example also leads to the conclusion that the dynamics of the photoionization is short range in nature and depends mostly on the size of the atom in its initial state.

\section{PHOTOIONIZATION OF TWO-ELECTRON ATOM}

For the multi-electron atoms (e.g., $\mathrm{H}^{-}, \mathrm{He}$ and $\mathrm{Li}$ ), a number of recent works [3, 6-8] appear to suggest that many of the singly excited states remain bound even when $D$ is smaller than the average size of an isolated atom in the same singly bound excited states. In particular, for the ground state of $H^{-}$, it concludes that even when $D$ is substantially smaller than the average size of the ground state of the isolated $H^{-}$, its ground state remains bound [7]. Such implication is intuitively and qualitatively different from the migration of the bound states into the continuum for the hydrogen atoms in plasma environment. This qualitative disparity between the disappearance of the bound states in one- and two-electron atoms may have resulted from an a priori application of the Debye screening to the electron-electron interaction in the form of

$$
V_{i j}=\frac{e^{2}}{r_{i j}} e^{-r_{i j} / D}
$$

where $r_{i j}$ is the distance between the atomic electrons. It has effectively led to a potential between the atomic electrons that is significantly more attractive than it should be by reducing greatly the Coulomb repulsion between atomic electrons, thus, leaving the atom in a bound state even when $D$ is smaller than the average size of an isolated atom in that particular bound state.

The less attractive nature of the screened Coulomb potential for an atomic electron in the field of the nuclear charge $Z$ could be understood easily since close to the atomic nucleus, there is a non-negligible presence of the free-moving electrons from the plasma outside with their relatively high mobility. However, to justify a similar Coulomb screening between two atomic electrons would require an unlikely qualitative picture, i.e., a substantial presence of the positive ions between atomic electrons, in spite of the relatively low mobility for the much heavier ions. In addition, since the screened potentials discussed above are derived from Gauss' law by assuming a stationary nuclear charge $Z$ located at $r=0$, a similar approach could not be applied directly to the free-moving atomic electrons. In other words, there is no compelling reason to modify the electronelectron interaction by the same Coulomb screening.

For the charged particle (e.g., proton) impact excitation of hydrogen-like atomic systems in plasma environment, the interaction between the incident particle and the atomic electron is long-range in nature either before or after the collision. Scheibner et al [22] proposed two different static potentials to treat such processes for 1) the low 
temperature and high density plasmas and 2) the high temperature and low density plasmas, respectively. The Debye screening in their calculation was included only between the incident charged particle and the target nucleus. Since the dynamics of the atomic photoionization is essentially short range in nature, together with the lack of theoretical ground to include the Debye screening in the electron-electron interaction between atomic electrons as we discussed earlier, the N-electron Hamiltonian for an atom in plasma environment in our photoionization calculation is expressed in terms of $h_{o}(r ; D)$ given in Eq. (1) as

$$
H\left(r_{i}, r_{j}, \cdots ; D\right)=\sum_{i=1, N} h_{o}\left(r_{i} ; D\right)+\sum_{i>j}^{N} \frac{e^{2}}{r_{i j}},
$$

where $r_{i j}=\left|\overrightarrow{r_{i}}-\overrightarrow{r_{j}}\right|$ represents the separation between the atomic electrons $i$ and $j$. The one-particle Hamiltonian $h_{o}$ for the individual atomic electron is subject to a potential $V_{s}$ given either by the Yukawa potential or the one from Eq. (7). The numerical results presented below are calculated with the B-spline based configuration interaction (BSCI) method which has been applied successfully to a large number of atomic structure properties. Details of the theoretical approach, the computation procedures, and a large number of its applications have already been presented elsewhere [20, 21]. The initial and final states of the photoionization are calculated typically with a basis set of atomic orbitals representing over 10,000 two-electron configurations. The calculated photoionization cross sections in length and velocity approximations agree to at least three digits or better. One of the advantages of the BSCI approach is its ability to map out the configuration mixed effective one-electron wavefunction $\xi_{\ell}(r)$ (see, Eq. (50) of [20]) of the photoionized outgoing electron. By examining explicitly this effective oneelectron outgoing wavefunction, we are able to analyze qualitatively the general feature of the photoionization spectra for the two-electron atoms similar to what we did earlier for the hydrogen atom.

For $H^{-}$, with only one bound state, the migration of the bound-bound transition into narrow resonance-like structure in the continuum near the ionization threshold is not expected. Indeed, Fig. 4 shows the absence of such resonance-like structure as $D$ decreases from the plasma-free $H^{-}$at $D=\infty$. The top plot in Fig. 4 presents the calculated spectra using the Yukawa potential and the bottom plot shows the ones using the potential given by Eq. (7) with a nominally assigned value of $A=17 a_{o}$ (i.e., about the size of $H^{-}$in its ground state). Except for minor quantitative difference, the general feature is nearly the same on how the spectra evolve as D varies. A careful inspection of the spectra indicates a near zero cross section for photoelectron with kinetic energy up to about $0.075 R y$ (i.e., about $1 \mathrm{eV}$ ) for the spectrum with $D=35 a_{o}$. Even at greater value of $D$, e.g., at $D=100 a_{o}$, the cross section remains near zero from the threshold until the kinetic energy of the photoelectron increases to about $0.35 \mathrm{eV}$. 
Figure 5 presents the configuration mixed effective wavefunctions $\xi_{p}$ for the outgoing photoionized $p$ electron at a number of momenta. The top plot shows that at $D=35 a_{o}$ the first local maximum of $\xi_{p}$ shifts outwards at a fairly rapid rate as the momentum $k$ decreases. In fact, at $k=0.32534$ (i.e., at a photoelectron energy about $1.4 \mathrm{eV}$ ), the first local maximum is already located at $r$ greater than $30 a_{o}$, i.e., at a distance from the origin at about twice the size of the $\mathrm{H}^{-}$in its ground state. In other words, the overlap between the wavefunctions of the outgoing electron and the electron in its initial state is relatively small. Clearly, the cross section, which is proportional to the square of the dipole matrix element, would decrease even faster as the overlap of the wavefunctions decreases rapidly when the momentum decreases, thus, a near zero cross section at an energy less than $1 \mathrm{eV}$ as shown. On the other hand, the penetration of the ionized electron to the smaller $r$ region also increases substantially at slightly higher momentum, thus a fast rise in cross section at energy higher than $1 \mathrm{eV}$. With a substantially less Debye screening at $D=100 a_{o}$, the bottom plot of Fig. 5 shows that the penetration of the outgoing electron to the small $r$ region is stronger than the ones at $D=35 a_{o}$, even when the photoelectron is less energetic. Consequently, the onset of the sharp rise in photoionization cross section occurs much closer to the threshold at around $0.35 \mathrm{eV}$. This threshold feature of the photoionization spectra is very different from that for the hydrogen atom subject to plasma environment, where the photoionization cross section starts its sharp increase at the threshold without any shift.

Qualitatively, this difference in the threshold feature could be accounted for by the presence of the second atomic electron in $H^{-}$. The presence of the remaining electron in the photoionization of $H^{-}$has the effect of reducing the probability density of the low energy outgoing electron in the small $r$ region due to the Coulomb repulsion between electrons. Since the screened Coulomb potential has the effect of increasing the average radius of the atomic orbit of the remaining electron, the reduction of the probability density of the ionized electron at small $r$ would be greater with a smaller $D$. Therefore, it requires more kinetic energy of the outgoing electron to penetration into the inner region of the atom for a more appreciable overlap between the initial and final orbits. This is exactly what happens when the sharp rise of the photoionization spectrum from nearzero cross section occurs at around $0.35 \mathrm{eV}$ with $D=100 a_{o}$, whereas with $D=35 a_{o}$, it starts at around $1 \mathrm{eV}$.

For $\mathrm{He}$ atom, with its two electrons and a large number of strong bound-bound transitions similar to the hydrogen atom, one could readily conclude that its photoionization spectra would have the general features similar to the ones we discussed earlier. Fig. 6 shows our theoretical results of the migration of the photoionization spectra corresponding to the $1 s^{2}{ }^{1} S \rightarrow 1 s 2 p{ }^{1} P$ transition with decreasing Debye length from 
$10 a_{o}$ to $6 a_{o}$. Indeed, the change from the narrow resonance-like structures into broad continuum spectra is exactly like those in the photoionization of hydrogen atom. Similar to the $\mathrm{H}^{-}$, the near-zero cross sections from the ionization threshold to a critical value of kinetic energy before the onset of fast rising photoionization cross section is also clearly seen. Figure 7 presents the variation of the effective one-electron wavefunctions $\xi_{p}$ and their quasi-bound features for the outgoing ionized electron near the peak of the resonance-like structure with energies between $0.0601 R y$ and $0.0613 R y$ at $D=10 a_{o}$. We should point out that the amplitude of the outside oscillating part of the radial wavefunction is noticeably smaller at $\epsilon=0.0606 R y$ when the cross section is at its peak value. Although it appears that the resonance-like structure is similar to the usual Fano-Beutler autoionization resonance in the photoionization of the plasma-free atoms, its nature is, nevertheless, different. The standard Fano resonance is primarily due to the configuration interaction between the doubly excited bound state and the singly excited ionized channel [23]. In other words, it is the configuration mixing between two different types of two-electron configurations with different angular momentum combinations, i.e., different $\ell_{1} \ell_{2}$. The resonance-like structure in the photoionization of atom in the plasma environment, on the other hand, is due to the mixing of bound and continuum components of the same angular momentum combination. Instead of a state function with the doubly excited bound component embedded in the singly ionized component with different $\ell_{1} \ell_{2}$ combination for the usual Fano resonance, what we have now is a state function with the singly excited quasi-bound component embedded in the singly ionized continuum component with the same $\ell_{1} \ell_{2}$ combination.

Figure 8 presents our theoretical results for $H e$ photoionization using the potential $V_{s}$ by Eq. (7) with a nominally chosen $A=4 a_{o}$. The vertical axis of the top plot is in $\log$ scale and the same spectra with the linear scale are shown in the bottom plot. Qualitatively, there is little difference in their general features compared to the ones shown in Fig. 6 with $A=0$, except the values of $D$ are chosen to be slightly smaller since the potential given by Eq. (7) is more attractive at $r \leq A$ for the same $D$. With $D=9 a_{o}$, the top plot of Fig. 9 shows that the magnitude of the inner most loop of the quasi-bound wavefunction of the outgoing electron at small $r$ rises up and falls down rapidly as energy changes by less than $0.0004 R y$ with a center maximum at around $k=0.22053$. It is interesting to note that the bottom plot of Fig. 9 shows a nearzero magnitude of the inner most loop of the wavefunctions of the outgoing electron at momenta on both side but further away from $k=0.22053$, due to the Coulomb repulsion from the remaining atomic electron as we discussed above. Since the photoionization cross section depends primarily on the overlap between the wavefunctions of the outgoing electron and the electron in its initial state, one could easily conclude, first, a narrow resonance-like spectrum and second, near-zero cross sections from threshold until the quasi-bound nature appears in the wavefunction of the outgoing electron. 


\section{CONCLUSION}

We present in this paper a detailed study of the photoionization of one- and twoelectron atoms in changing plasma environment based on the Debye-Hückel model. We have examined a number of general features of the photoionization spectra, including the common ones and also the distinctly different ones. For the one-electron hydrogenic system, we are able to link the photoionization spectra to the overlap between the wavefunctions of the outgoing ionized electron and the electron in its initial orbit, or, more specifically the dipole matrix for the transition. Since the atomic orbit of the electron in its initial state is usually limited to small $r$ region, the dynamics of the atomic photoionization from its ground state is short-ranged in nature. For the two-electron atoms, we are able to carry out similar analysis based on the configuration-mixed effective one-electron wavefunctions using the B-spline based approach. We have concluded that the plasma-induced narrow resonance structures near the ionization threshold as the bound excited atomic states migrate into the continuum are due to the configuration mixing of quasi-bound and continuum components of the same $\ell_{1} \ell_{2}$ angular momentum combination in the state wavefunction. In other words, they are different in nature from the Fano-Beutler resonance, which is due to a state function with the doubly excited bound component embedded in the singly ionized component with different $\ell_{1} \ell_{2}$ combination. In addition, our calculation has led to a small but finite energy region with near-zero cross section starting from the ionization threshold in two-electron spectra. This near-zero cross section window could be directly attributed to the presence of the second electron in the two-electron atoms. Finally, we would like to point out that the red-shifted narrow absorption resonances with large peak cross section such as the one shown in the bottom plot of Fig. 1 may offer the opportunity for observation with sufficient initial state population and the currently available highly intense continuous light sources for the laser-produced plasmas similar to those already realized [17, 18].

\section{ACKNOWLEDGMENTS}

This work was supported by the National Science Council in Taiwan under the grant nos. NSC 99-2112-M-030-003 and NSC102-2119-M-007-003. One of us (TNC) wishes to acknowledge a few in-depth discussions with Werner Däppen. 


\section{References}

[1] A. N. Sil and P. K. Mukherjee, Int. J. Quantum. Chem. 102, 1061 (2005).

[2] P. K. Mukherjee, J. Karwowski, and G. H. F. Diercksen, Chem. Phys. Lett. 363, 323 (2002).

[3] H. Okutsu, T. Sako, K. Yamanouchi, and G. H. F. Diercksen, J. Phys. B38, 917 (2005).

[4] S. B. Zhang, J. G. Wang, and R. K. Janev, Phys. Rev. A81, 032707 (2010); Phys. Rev. Lett. 104, 023203 (2010); Y. Y. Qi, Y. Wu, J. G. Wang, and Y. Z. Qu, Phys. Plasmas 16, 023502 (2009).

[5] C. Y. Lin and Y. K. Ho, Comp. Phys. Comm. 182, 125 (2011); C. Y. Lin and Y. K. Ho, Eur. Phys. J. D57, 21 (2010).

[6] S. Sahoo and Y. K. Ho, J. Quant. Spectrosc. Radiat. Trans. 111, 52 (2010); S. Kar and Y. K. Ho, J. Quant. Spectrosc. Radiat. Trans. 109, 445 (2008); S. Kar and Y. K. Ho, Phys. Plasmas 15, 013301 (2008); S. Kar and Y. K. Ho, Int. J. Quantum Chem. 106, 814 (2006); A. Ghoshal and Y. K. Ho, J. Phys. B42, 075002 (2009).

[7] A. Ghoshal and Y. K. Ho, J. Phys. B42, 175006 (2009).

[8] Z. Wang and P. Winkler, Phys. Rev. A52, 216 (1995).

[9] S. T. Dai, A. Solovyova, and P. Winkler, Phys. Rev. E64, 016408 (2001).

[10] X. Lopez, C. Sarasola, and J. M. Ugalde, J. Phys. Chem. A101, 1804 (1997).

[11] F. J. Rogers, H. C. Graboske, Jr., and D. J. Harwood, Phys. Rev. A 1, 1577 (1970).

[12] C. A. Rouse, Phys. Rev. 163, 62 (1967).

[13] H. Margenau and M. Lewis, Rev. Mod. Phys. 31, 569 (1959).

[14] P. Debye and E. Hückel, Phyzik Z. 24, 185 (1923).

[15] W. Däppen, Astrophys. Space Sci., 328, 139 (2010).

[16] W. Däppen, J. Phys. A39, 4441 (2006); in Solar and Stellar Physics Through Eclipses, edited by Osman Demircan, Selim O. Selam and Berahitdin Albayrak (Astronomical Society of the Pacific Conference Series 370, 2007), p. 3-12.

[17] Y. Leng, J. Goldhar, H. R. Griem, and R. W. Lee, Phys. Rev. E52, 4328 (1995). 
[18] A. Saemann et al, Phys. Rev. Lett. 82, 4843 (1999); R. C. Elton, J. Ghosh, H. R. Griem, and E. J. Iglesias, Phys. Rev. E69, 067403 (2004).

[19] G. V. Marr, in Photoionization Processes in Gases (Acad. Press; NY, 1967), p. 110.

[20] T. N. Chang, in Many-body Theory of Atomic Structure and Photoionization, edited by T. N. Chang (World Scientific, Singapore, 1993), p. 213.

[21] T. N. Chang and T. K. Fang, Radiat. Phys. Chem. 70, 173 (2004); T. N. Chang and T. K. Fang, Phys. Rev. A52, 2638 (1995); T. N. Chang and X. Tang, Phys. Rev. A44, 232 (1991); T. N. Chang, Phys. Rev. A39, 4946 (1989).

[22] K. Scheibner, J. C. Weisheit and, N. F. Land, Phys. Rev. A35, 1252 (1987).

[23] U. Fano, Phys. Rev. 124, 1866 (1961). 
Figure 1: Hydrogen photoionization spectra corresopnding to $1 s \rightarrow 4 p$ transition as $D$ varies with $A=0$.

Figure 2: The radial wavefunctions $\chi_{k p}$ of the outgoing ionized electron of the plasma-free hydrogen at a number of momenta. The bottom plot compares $\chi_{k p}$ with $\chi_{1 s}$ wavefunction (reduced by a factor of 10 ) of the initial $1 s$ orbit at small $r$.

Figure 3: The radial wavefunction $\chi_{k p}$ of the outgoing ionized electron of hydrogen with $D=24.5 a_{o}$ at a number of momenta.

Figure 4: $H^{-}$photoionization spectra with $A=0$ and $A=17 a_{o}$ as $\mathrm{D}$ varies.

Figure 5: The effective radial wavefunctions $\xi_{p}$ of the outgoing ionized electron of $H^{-}$with $D=35 a_{o}$ and $D=100 a_{o}$ at a number of momenta.

Figure 6: He photoionization spectra corresopnding to $1 s^{2}{ }^{1} S \rightarrow 1 s 2 p{ }^{1} P$ transition as $D$ varies with $A=0$.

Figure 7: The variation of the effective radial wavefunctions $\xi_{p}$ of the outgoing ionized electron of $H e$ near the peak of the resonance-like structure with $A=0$ and $D=10 a_{o}$.

Figure 8: He photoionization spectra corresponding to $1 s^{2}{ }^{1} S \rightarrow 1 s 2 p{ }^{1} \mathrm{P}$ transition with $A=4 a_{o}$ as $D$ varies.

Figure 9: The variation of the effective radial wavefunctions $\xi_{p}$ of the outgoing ionized electron of He near the peak of the resonance-like structure with $D=9 a_{o}$ and $A=4 a_{o}$. 

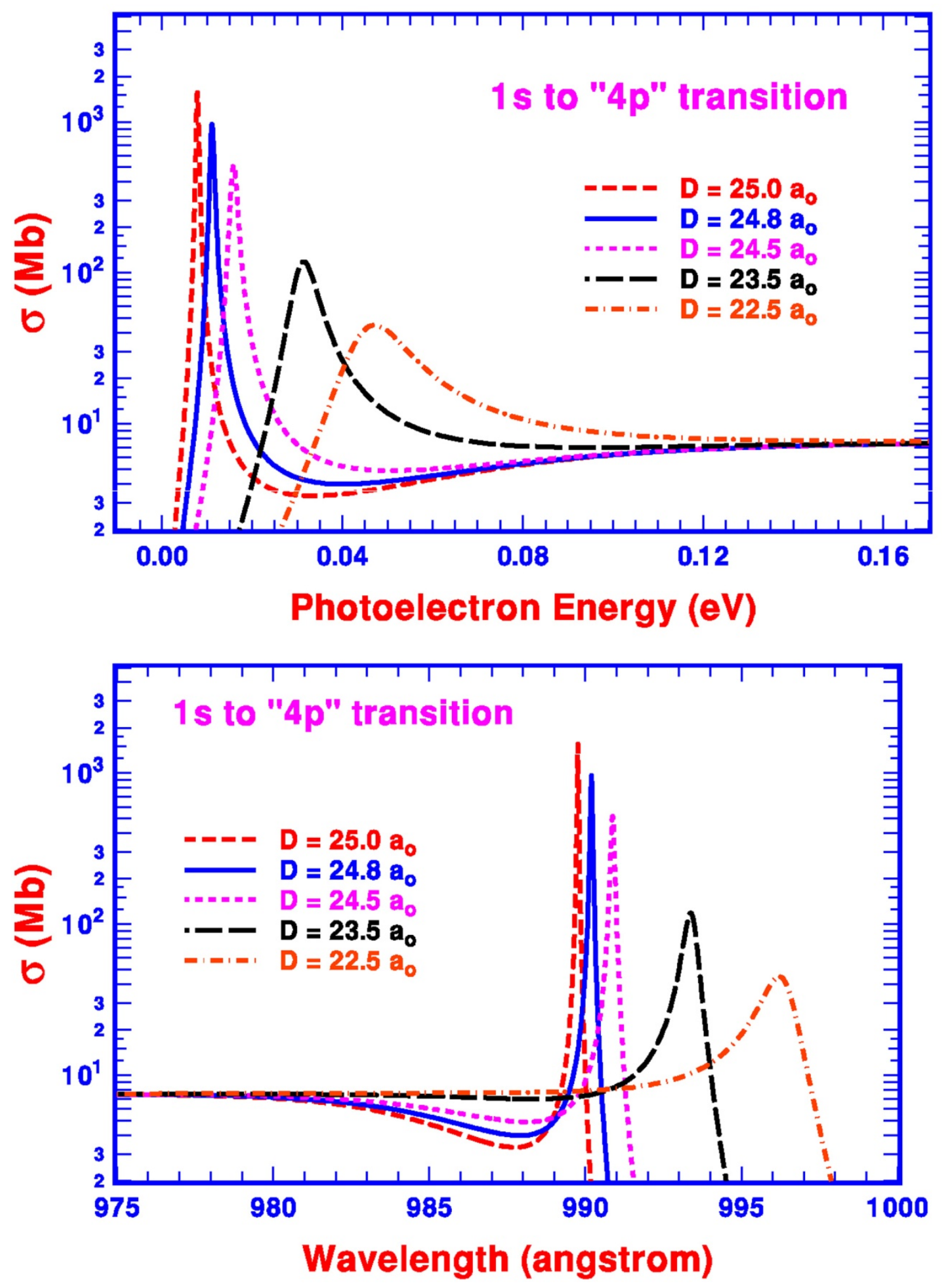

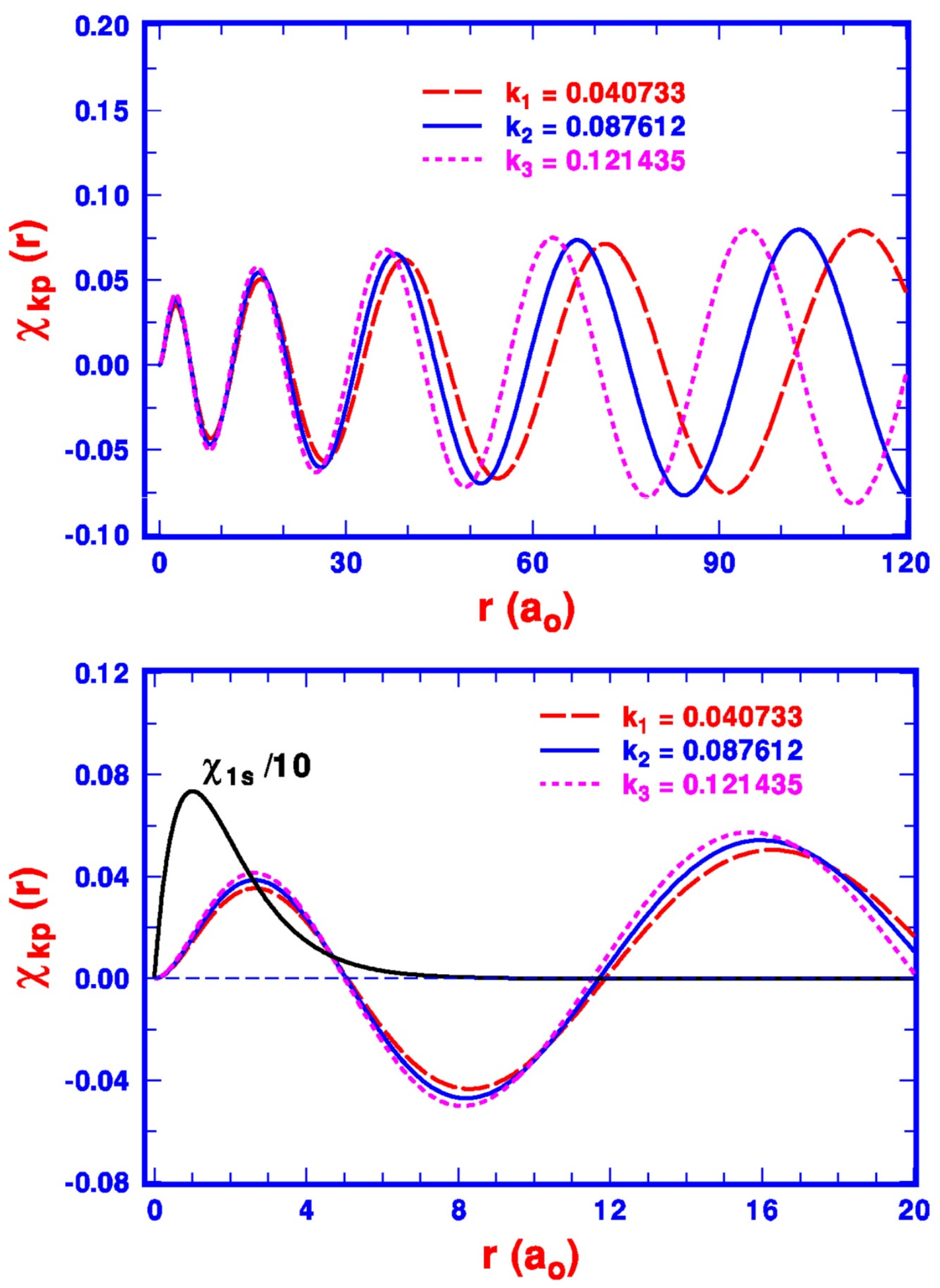


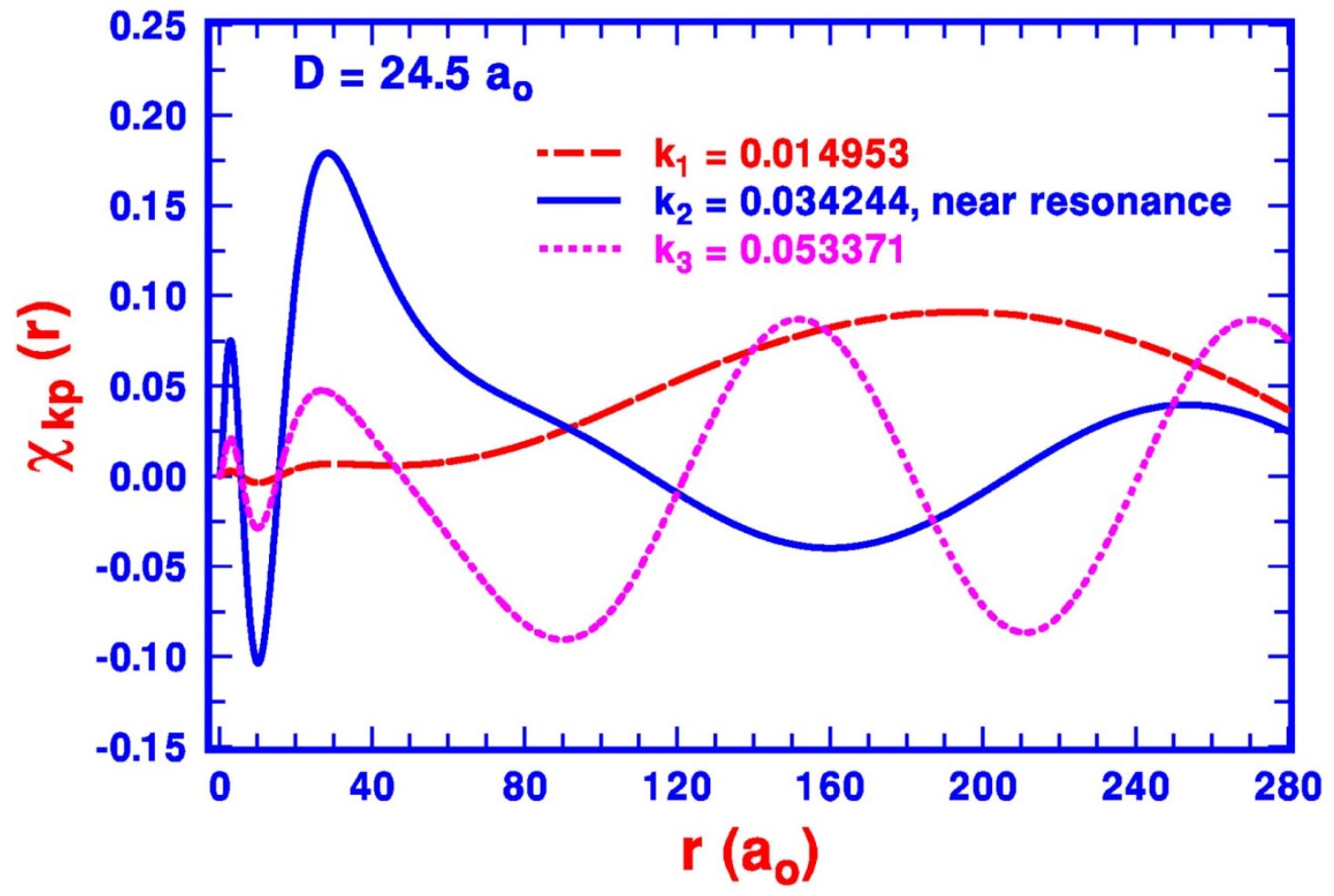



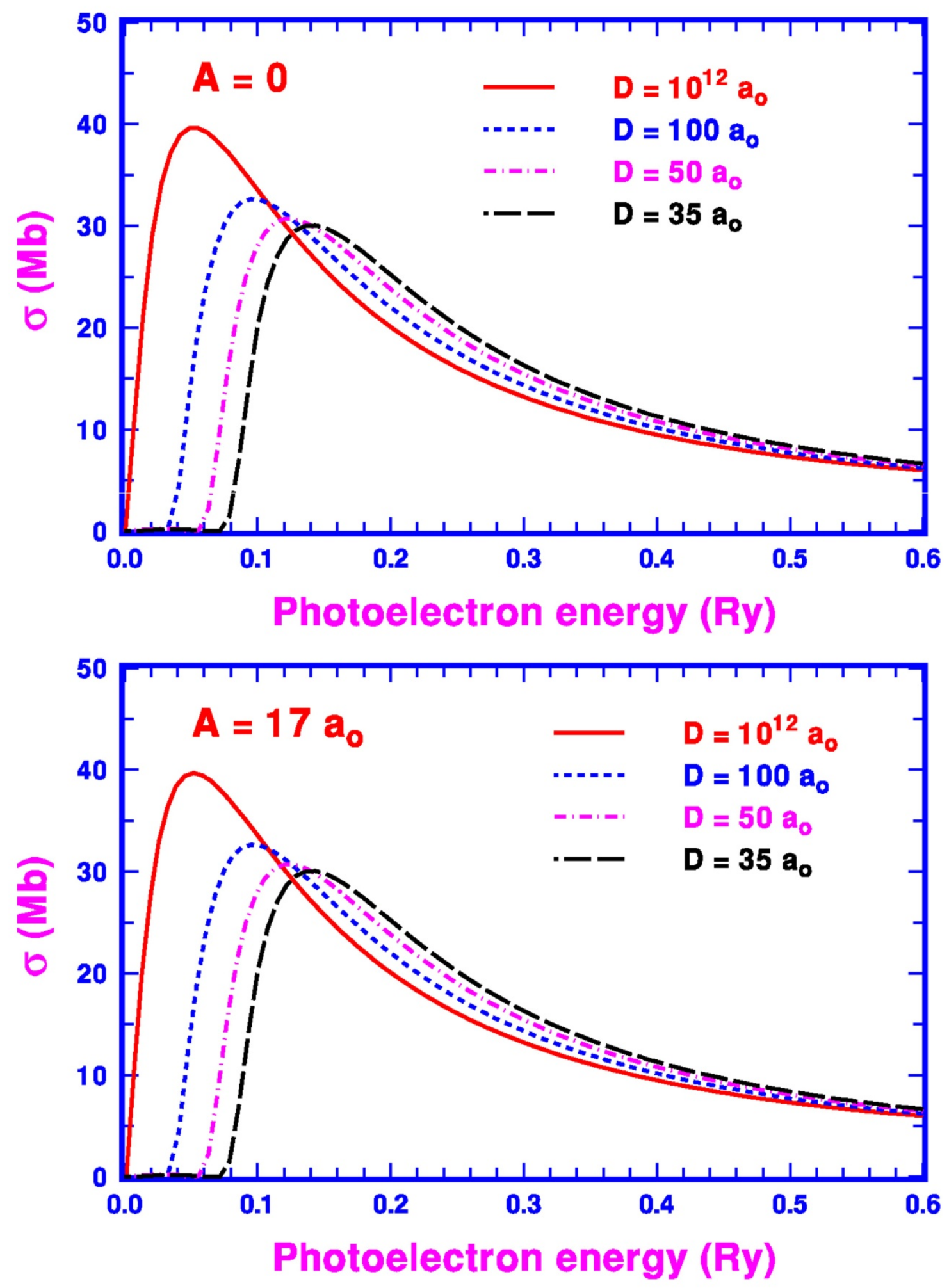

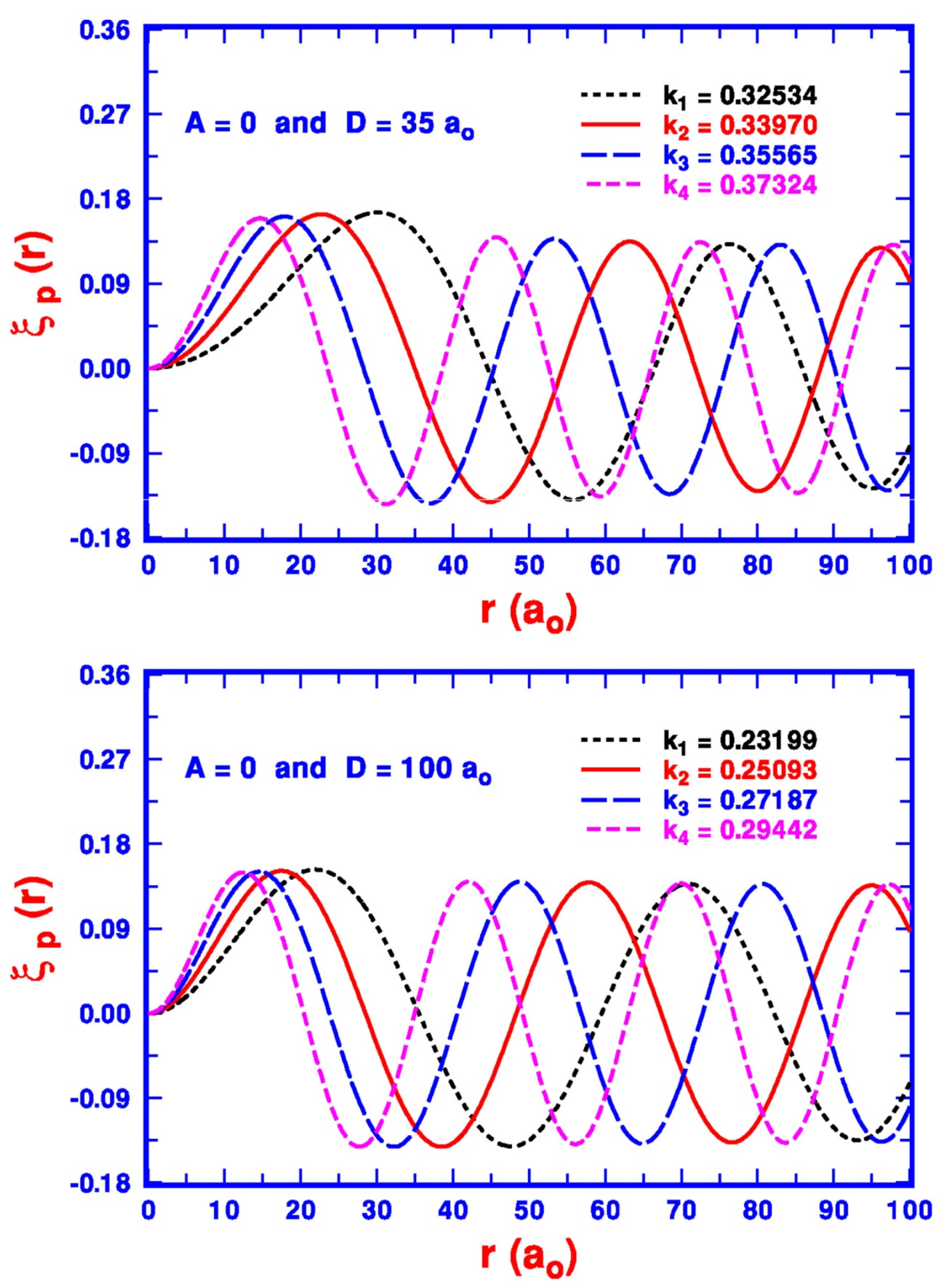


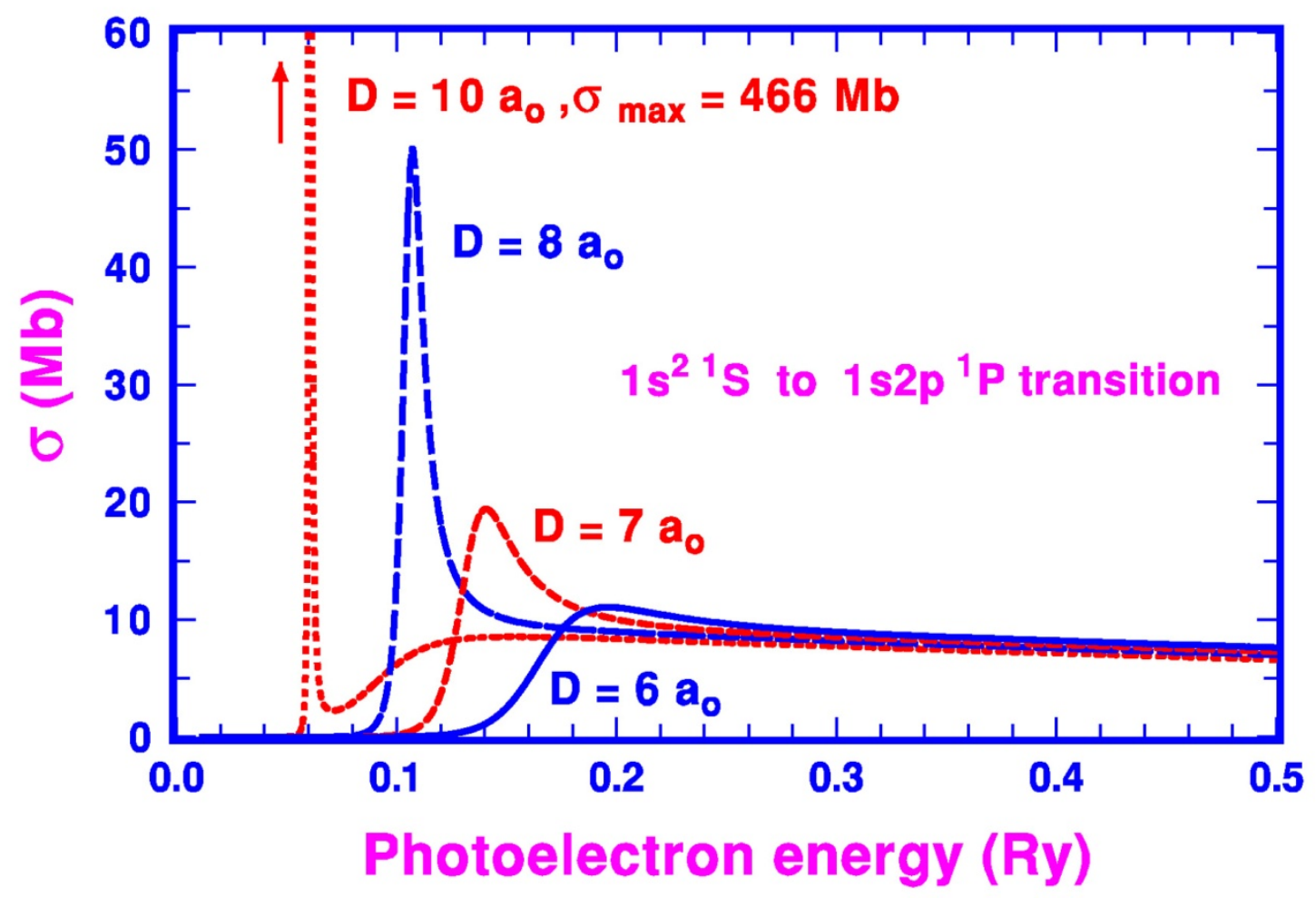



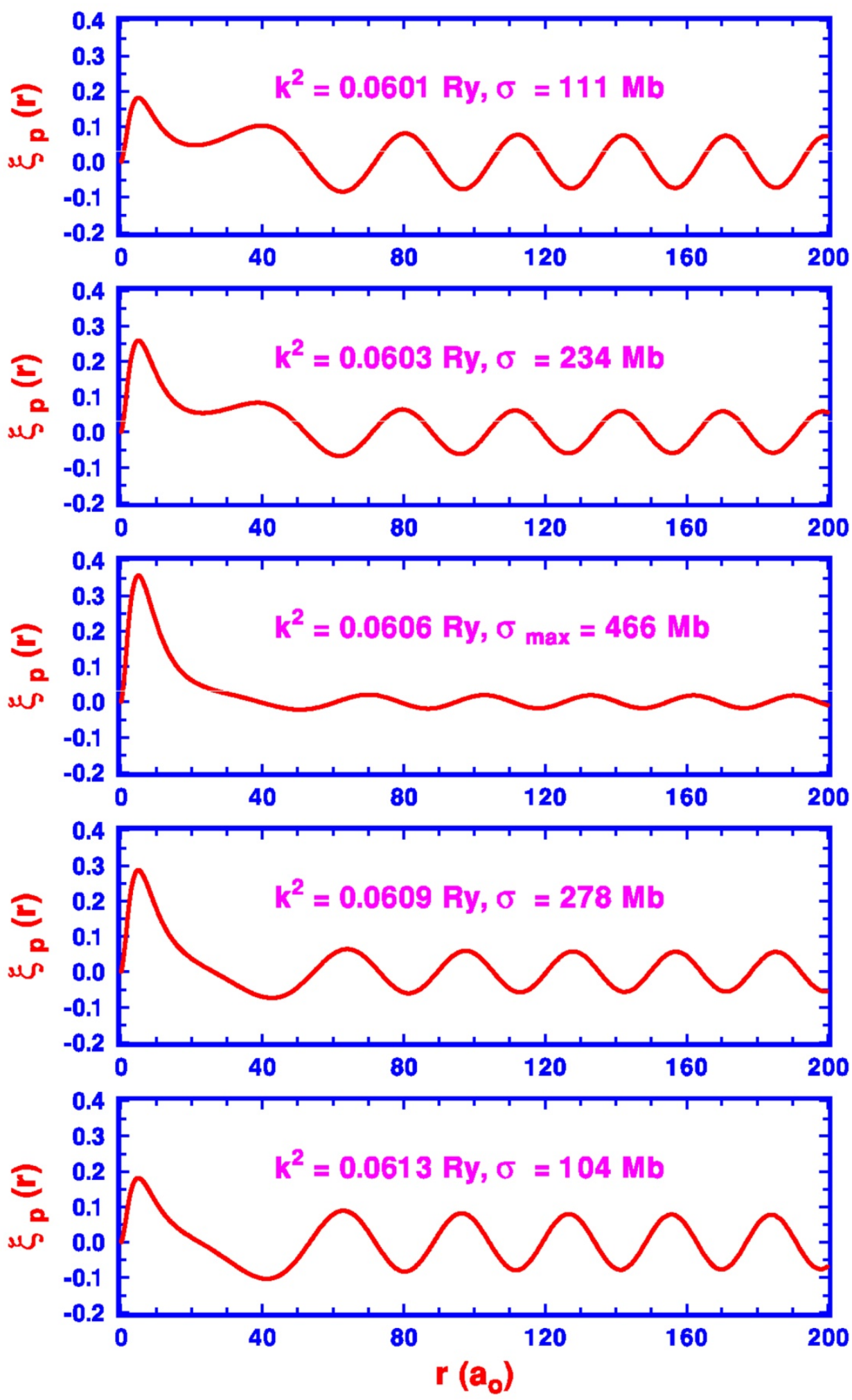

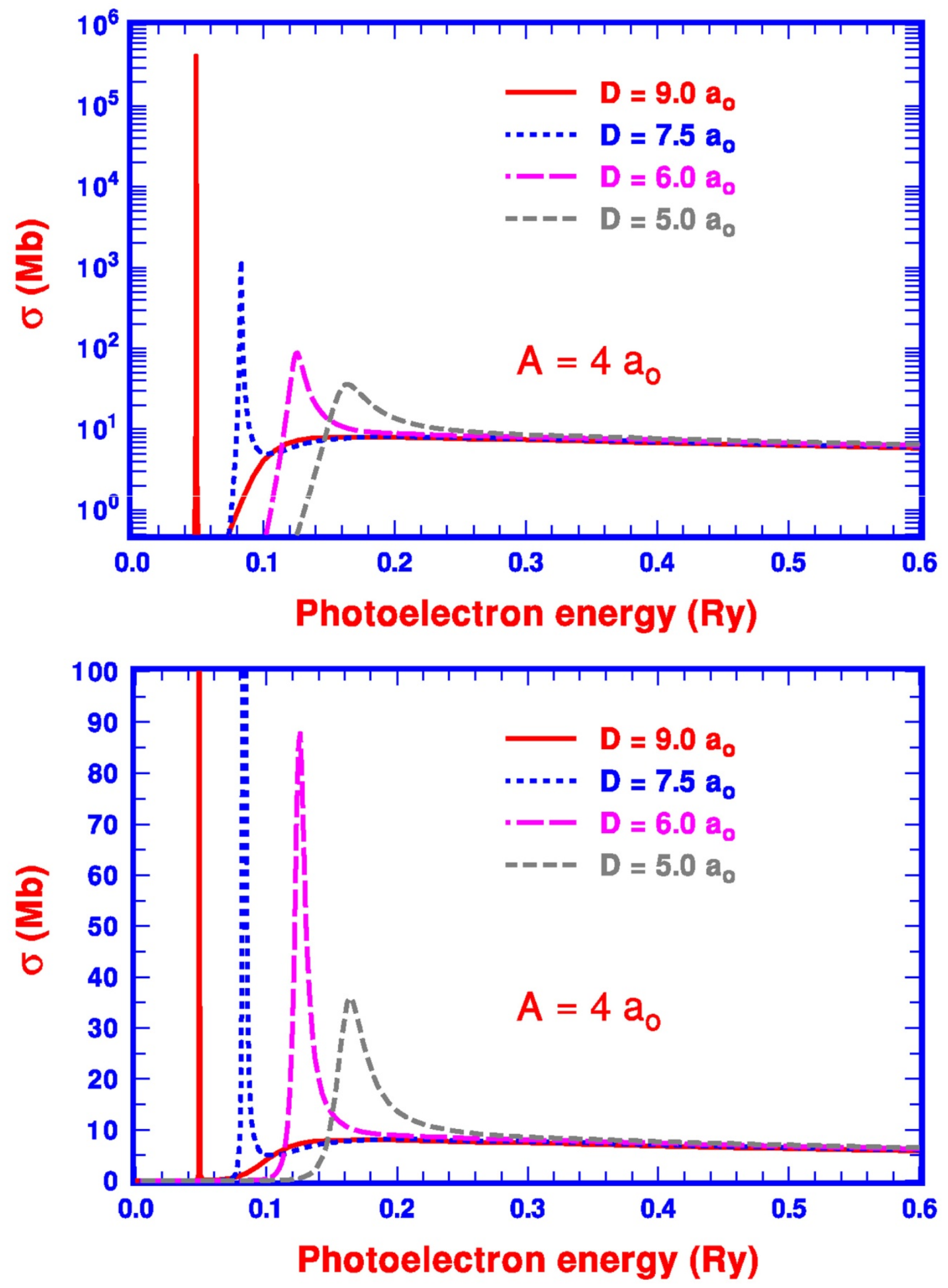

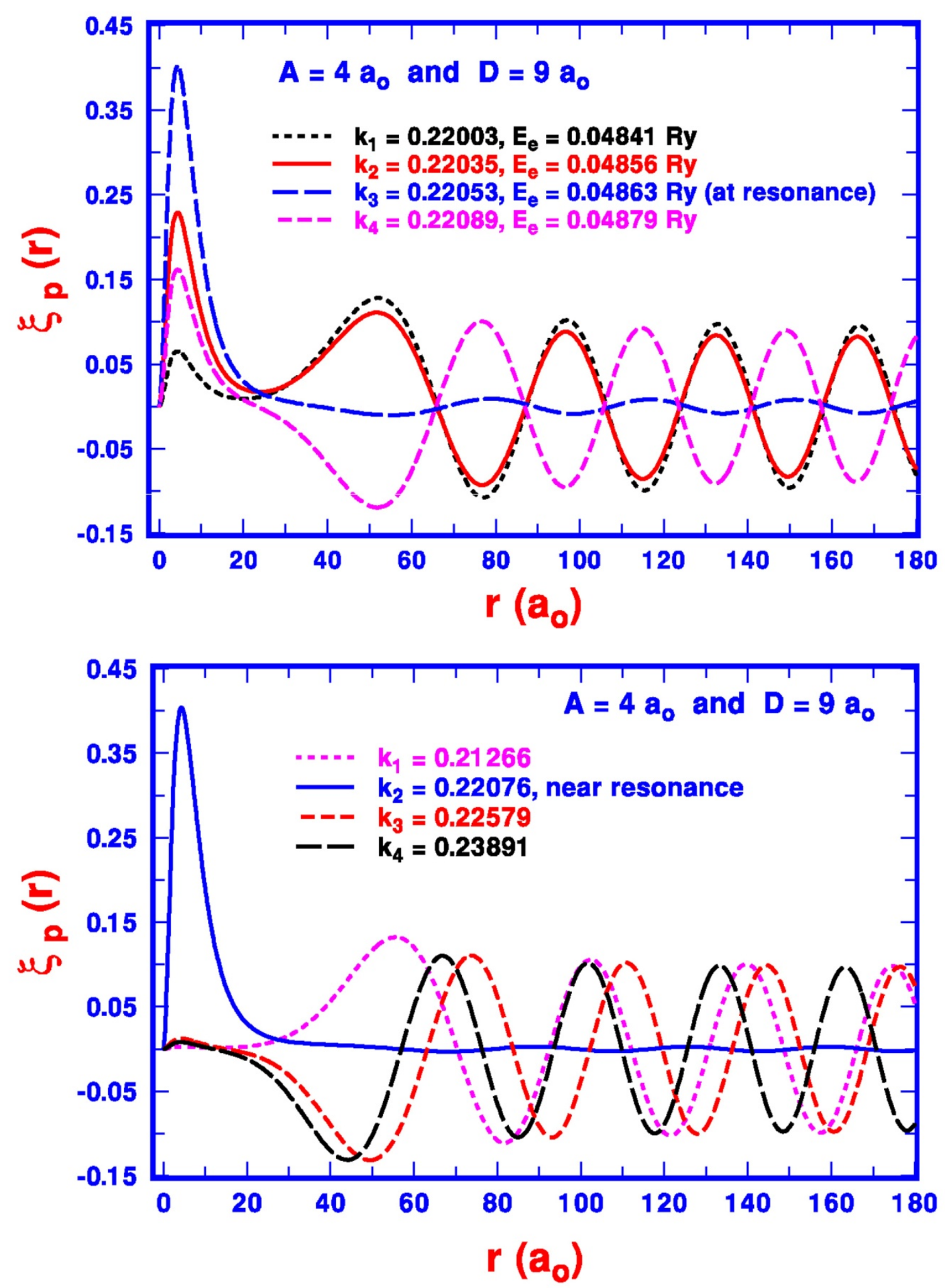